\title{
Analysis and Modeling of Physical Stratum for Power Line Communication
}

\author{
Prof. Madhuri Zambre \\ Professor, Department of Electrical Engineering, Modern College of Engineering Pune India \\ madhurizambre01@gmail.com
}

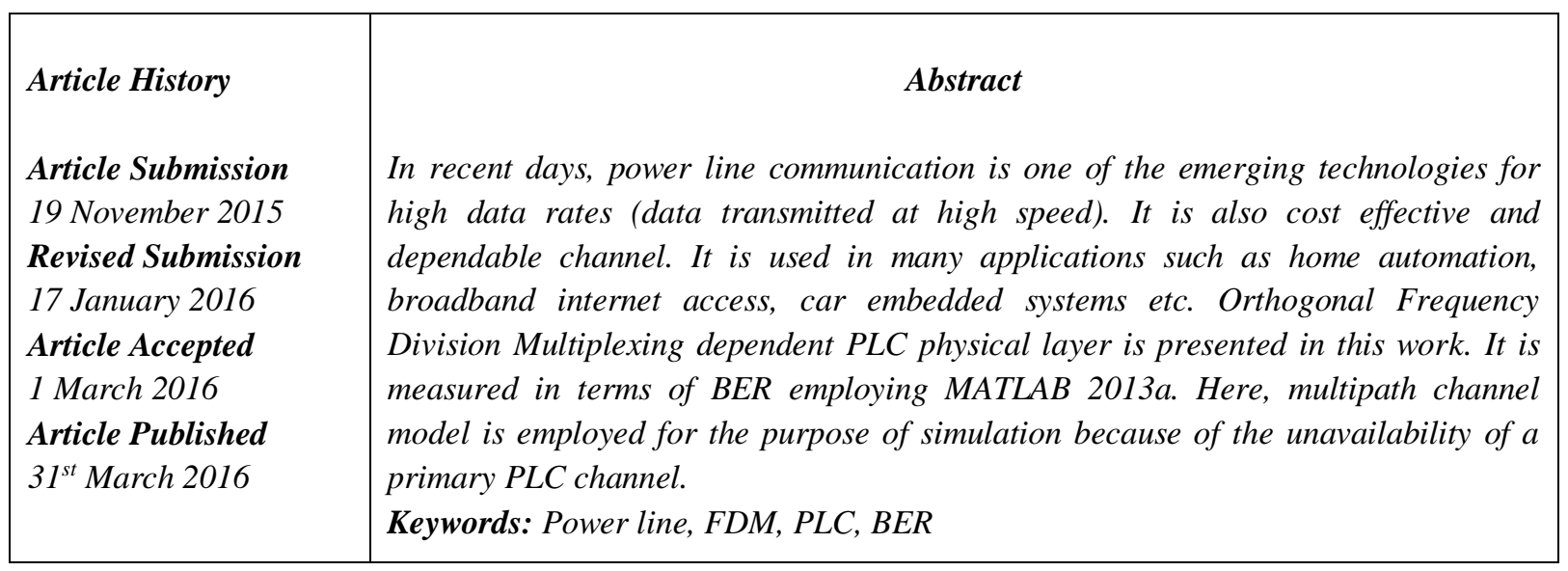

\section{Introduction}

In recent years, power line communication is becoming more and more popular in wireless communications because of the usage of known network power link. These power lines are risk communication channel because of fading, attenuation, noise, propagation by using multipath [1]. Power line noises are identified based on the mixture of narrowband noise, noise of impulses, featured noise. To install a dependable high data rate PLC system, power link characteristics and mismatch in the impedance causing multipath propagation are kept in mind. Between $1-30 \mathrm{MHz}$ is the frequency range that is taken into consideration for the functioning of a PLC system. In a PLC system, we go for an important modulation technique which is known as Orthogonal Frequency Division Multiplexing [2]. It divides the whole spectrum into many orthogonal subcarriers which are closely- spaced that engages a narrower bandwidth in comparison with any single carrier modulation scheme. Therefore, by employing OFDM we can limit the cause of attenuation in the frequency selective method, effect of propagation by using multipath [3]. Also, in PLC, data signals that are transmitted will not follow a uni-path, instead they go for multipath same as mobile signals employed in cellular and mobile transmissions. The common method that is accepted worldwide that is taken into consideration for simulating the above signals is Zimmermann and Dostert's channel model [4].

\section{Related PLC channel models}

The PLC channel modeling is a challenging one due to impedance mismatch, attenuation and time-varying behavior. Apart from these, a PLC system suffers from colored background noise and impulsive noises. Measurement is also a challenge in channel modeling. Therefore, a deep analysis on channel characterizations is required for an effective and reliable channel model [5].

In developing a communication media, there are two approaches employed to handle the disturbances. They are top-down approach and bottom-up approach. Developing the behavioral model, channel's parameters are considered in the first method. Whereas in the second method, it have a physical layer that connects to the primary channel having low -level parameters [6][7]. There are many models identified in the research, there is no specific channel model exists in terms of results due to measurements, approximations and topologies of a network. Also, in PLC, data signals that are transmitted will not follow a uni-path, instead they go for multipath same as mobile signals employed in cellular and mobile transmissions. LV is a central transmission line where it 
consists of many branches that move to the position of users. $\mathrm{T}_{\mathrm{x}}$ represents transmission point, $R X$ represents receiver point. This is shown in figure 1.

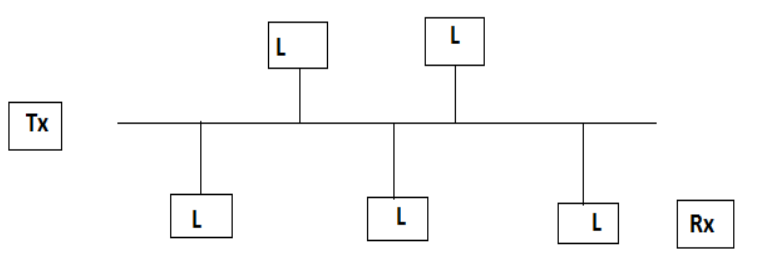

Fig 1: Schematic representation of transmission line in power grid

To assess propagation of multipath signal, we observe the below figure 2 .

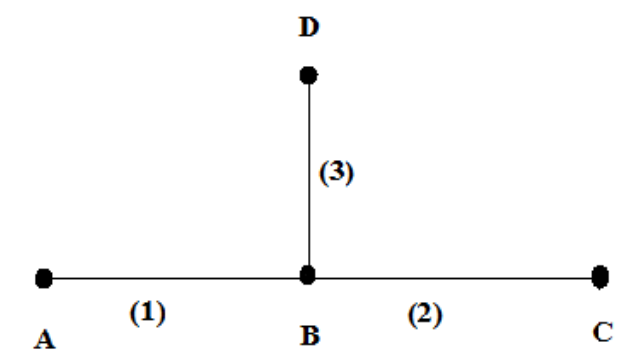

Fig 2: Schematic representation of propagation of multipath signal from $D$ to $C$

Here, we consider $\mathrm{D}$ is the transmission point, $\mathrm{C}$ is the receiving point. Near the point $\mathrm{D}$, produced signal should consider the following paths [8][9]. The above expression represents the propagation of a multipath signal. Followed path and the path length based received signal measures BER, power of the signal. Propagation of multipaths in PLC gives a delay represented by:

$$
\tau_{i}=\frac{d_{i} \sqrt{\varepsilon_{r}}}{c_{0}}=\frac{d_{i}}{\vartheta_{p}}
$$

$d_{i}$ Represents path length, $c_{0}$ represents light speed and $\varepsilon_{\mathrm{r}}$ represents dielectric constant.

$$
H(f)=\sum_{i=1}^{N} g_{i} \cdot A\left(f, d_{i}\right) \cdot e^{-j 2 \pi f \tau_{i}}
$$

Frequency response of the link between source and destination is given by $\mathrm{H}(\mathrm{f})$. If this grid network is becoming more and more and difficult, it should be divided into small sub-channels in studying them individually. $A\left(f, d_{i}\right)$ represents losses in the cable in terms of leakage or heat, operating frequency is $f, g_{i}$ represents weight component [10].

$$
g_{i} \leq 1
$$

Here, $g_{i}$ and $A\left(f, d_{i}\right)$ are the values that are experimentally identified. The above components models a mathematical PLC with multipath is presented.

$$
H(f)=\sum_{i=1}^{N} g_{i} \cdot A\left(f, d_{i}\right) \cdot e^{-j 2 \pi f \tau_{i}}
$$


After almost assessment done on $\mathrm{A}\left(\mathrm{f}, \mathrm{d}_{\mathrm{i}}\right)$, we go for assuming the mathematical formula for attenuation factor $(\alpha)$

$$
\alpha(f)=\alpha_{0}+\alpha_{1} \cdot f^{k}
$$

$\alpha_{0}$ and $\alpha_{1}$ are attenuation parameters leading to:

$$
A\left(f, d_{i}\right)=e^{-\alpha(f) \cdot d_{i}}=e^{-\left(\alpha_{0}+\alpha_{1} \cdot f^{k}\right) \cdot d_{i}}
$$

$A\left(f, d_{i}\right)$ is employed in $H(f)$ that offers modeling a channel fir transmission in the PLC link

$$
H(f)=\sum_{i=1}^{N} g_{i} \cdot e^{-\left(\alpha_{0}+\alpha_{1} \cdot f^{k}\right) \cdot d_{i}} \cdot e^{-j 2 \pi f \frac{d_{i}}{\vartheta_{p}}}
$$

$g_{i}=$ weighting factor

$e^{-\left(\alpha_{0}+\alpha_{1} \cdot f^{k}\right) \cdot d_{i}}=$ attenuation portion

$e^{-j 2 \pi f \frac{d_{i}}{\vartheta_{p}}}=$ delay portion

\section{Proposed Physical Layer for PLC}

Orthogonal Frequency Division Multiplexing is a specific modulation in multicarrier medium that is best suited for dispersive channel transmission. We here identifies many carriers which are orthogonal to one another, i.e., one is independent on another.

The transmitter section includes coding technique, interleaving, modulation used, pilot insertion, IFFT and cyclic prefix. Modulation is the one specific technique in which the transmission of signal from transmitter to receiver through a communication link that concludes in reducing the noise effect. Also, the received signal is demodulated to get back the initial signal. OFDM is a modulation technique that divides the high speed information into small sub carriers which orthogonal with one another. This result is attained by employing a modulation technique known as Quadrature Amplitude Modulation. Thereafter, IFFT is applied on the signal. Moreover, it is again passed via a converter for further processing.

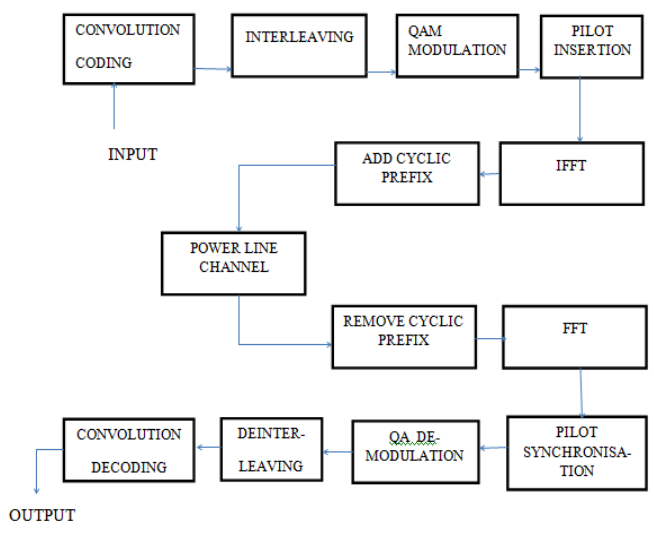

Fig 3: FFT-based physical layer for PLC 
In the first stage, OFDM information is converted from serial to parallel data. Thereafter, FFT bring digital samples into frequency samples. Hence, amplitudes of initial data are the frequency components. In the final stage, parallel data is converted into serial data to retrieve the initial data at the input.

\section{Simulation Results}

The above parameter is generated in terms of frequency components. Also, we know that the difference between calculation and simulation is very small. The impulse response takes the dominant paths that are satisfied by employing four-path model.

Table 1: Attenuation and path parameters for PLC channel model

\begin{tabular}{|c|c|c|c|c|c|}
\hline \multicolumn{6}{|c|}{ Attenuation parameters } \\
\hline \multicolumn{2}{|c|}{$\mathrm{K}=1$} & \multicolumn{2}{|c|}{$\alpha_{0}=0$} & \multicolumn{2}{|c|}{$\alpha_{1}=7.8 * 10^{-10} \mathrm{~s} / \mathrm{m}$} \\
\hline \multicolumn{6}{|c|}{ Path parameters } \\
\hline $\mathbf{i}$ & $\mathbf{g}_{\mathbf{i}}$ & $\mathbf{d i}_{\mathrm{i}} / \mathbf{m}$ & $\mathbf{i}$ & gi & $\mathbf{d i}_{\mathrm{i}} / \mathbf{m}$ \\
\hline 1 & 0.64 & 200 & 3 & -0.15 & 240.6 \\
\hline 2 & 0.38 & 222.4 & 4 & 0.05 & 260.2 \\
\hline
\end{tabular}

Based on the measured attenuation and path parameters, the coding is simulated. The respective output including gain and phase responses are obtained. The above components are generated in terms of frequency components. . Also, we know that the difference between calculation and simulation is very small. This is shown in figure 4.

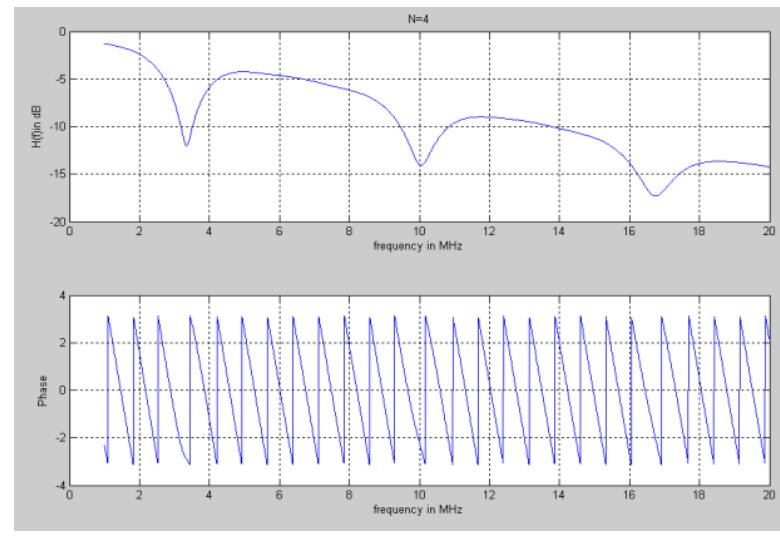

Fig 4: PLC channel model gain and phase plot, $N=4$

The impulse response paths are recovered to minimize the model during $\mathrm{N}=15$. Also, model measurements in frequency response displays disturbances at deep notched locations. The results are tabulated in table 2.

Table 2: Attenuation and path parameters for PLC channel model, $N=15$

\begin{tabular}{|c|c|c|c|c|c|}
\hline \multicolumn{6}{|c|}{ Attenuation parameters } \\
\hline \multicolumn{2}{|c|}{$\mathrm{K}=1$} & \multicolumn{2}{|c|}{$\alpha_{0}=0$} & \multicolumn{2}{|c|}{$\alpha_{1}=7.8 * 10^{-10} \mathrm{~s} / \mathrm{m}$} \\
\hline \multicolumn{6}{|c|}{ Path parameters } \\
\hline $\mathbf{i}$ & $\mathbf{g}_{\mathbf{i}}$ & $d_{i} / \mathbf{m}$ & $\mathbf{i}$ & gi & $\mathbf{d}_{\mathrm{i}} / \mathbf{m}$ \\
\hline 1 & 0.029 & 90 & 9 & -0.071 & 410 \\
\hline 2 & 0.0430 & 102 & 10 & -0.035 & 480 \\
\hline 3 & 0.0103 & 113 & 11 & 0.065 & 565 \\
\hline 4 & -0.058 & 143 & 12 & -0.055 & 720 \\
\hline
\end{tabular}




\begin{tabular}{|c|c|c|c|c|c|}
\hline 5 & -0.045 & 148 & 13 & 0.042 & 930 \\
\hline 6 & -0.040 & 200 & 14 & -0.059 & 1120 \\
\hline 7 & 0.038 & 230 & 15 & 0.049 & 1200 \\
\hline 8 & -0.038 & 322 & & & \\
\hline
\end{tabular}

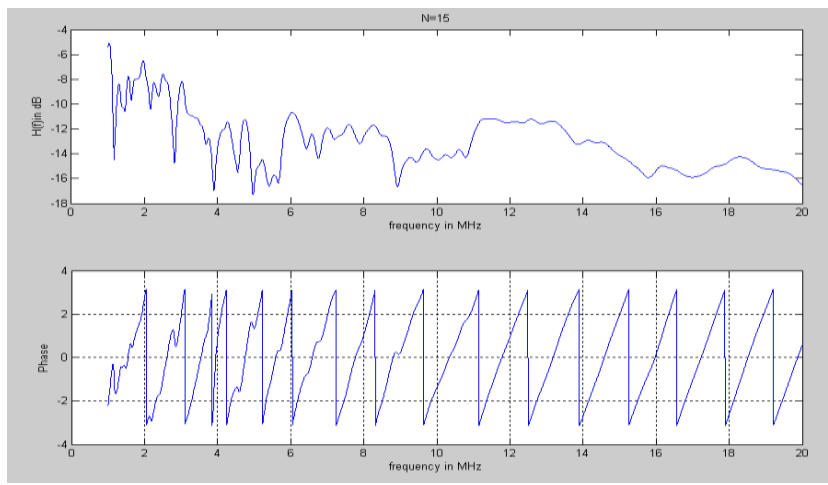

Fig 5: PLC channel model gain and phase plot, $N=15$

BER performance is calculated for the developed transceiver above PLC by employing MATLAB R2013a where the simulation of response of the receiver is observed in the below figure 6 .

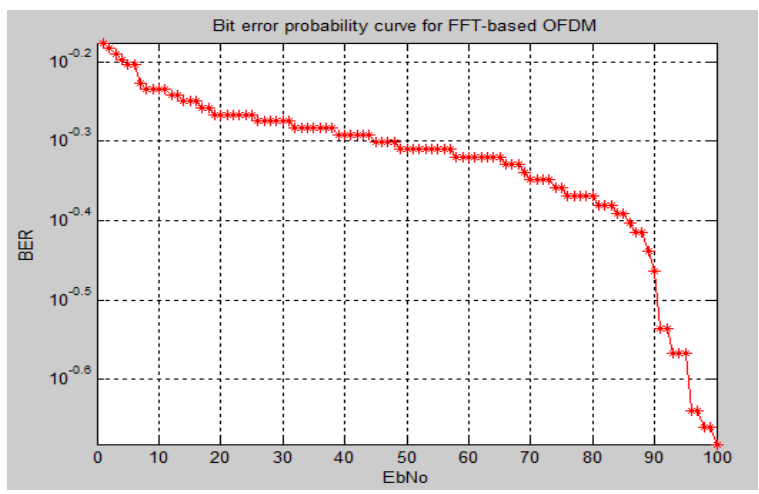

Fig 6: BER curve for FFT-based PLC

\section{Conclusion}

The important aspect of this paper is in designing an efficient physical layer for power line communication. Performance is evaluated by employing the system using both combination of FFT and QAM are simulated in MatlabR2013a. In future, the project is extended to reduce bit error rate further by combinations of different modulation techniques and error-correcting codes. And also to improve the efficient transmission capacity as much as possible.

\section{References}

[1] Ferreira, H.C., Lampe, L., Newbury, J. and Swart,T.G. (2010) Power line Communications: Theory and Applica tions for Narrowband and Broadband Communications over Power Lines. Wiley.

[2] Zimmermann, M. and Dostert, K. (2002) Analysis and Modeling of Impulsive Noise in Broad-Band Powerline Communications. Electromagnetic Compatibility, IEEE Transactions on Electromagnetic Compatibility, 44, 249-258.

[3] Gotz, M., Rapp, M. and Dostert, K. (2004) Power Line Channel Characteristics and Their Effect on Communication System Design. IEEE Communications Magazine, 42, 78-86. 
[4] Pavlidou, N., Han Vinck, A., Yazdani, J. and Honary, B. (2003) Power Line Communications: State of the Art and Future Trends. IEEE Communications Magazine, 41, 34-40.

[5] Zimmermann M. and Dostert, K. (2002) A Multipath Model for the Power-Line Channel. IEEE Transactions on Communications, 50, 553-559.

[6] Meng.H , Chen.S, Guan.Y, Law.C, So.P, Gunawan.E, Lie.T (2004), 'Modeling of Transfer Characteristics for the Broadband Power Line Communication Channel', IEEE Transactions on Power Delivery 19 (3) 1057-1064.

[7] Petr Mllynek,Jiri Miisurec,Martin Koutny,Milos Orgon (2011), 'Power line cable transfer function for modelling of power line communication system', Journal of Electrical Engineering, Vol. 62, No. 2,104-108.

[8] IEEE Draft Standard for the Requirements for Power Line Carrier Line Traps," in IEEE PC93.3/D16.1, August 2015 , vol., no., pp.1-40, 1 Jan. 2015.

[9] T. N. Vo, K. Amis, T. Chonavel and P. Siohan, "Guard interval adaptation for in-home power line communication," 2015 IEEE International Symposium on Power Line Communications and Its Applications (ISPLC), Austin, TX, 2015, pp. 245-249, doi: 10.1109/ISPLC.2015.7147622.

[10] S. Barmada, P. Mezzanotte, L. Roselli and M. Tucci, "Full simulation of a wireless power transfer system with power line communication integration," 2015 31st International Review of Progress in Applied Computational Electromagnetics (ACES), Williamsburg, VA, 2015, pp. 1-2. 